\section{Microscopy and Microanalysis of Nano-Scale Materials}

J. R. Michael, L. N. Brewer, D. C. Miller, K. R. Zavadil,

S. V. Prasad and P. G. Kotula

Materials Science Center

Sandia National Laboratories, Albuquerque, NM

jrmicha@sandia.gov

\section{Introduction}

Material scientists and engineers continue to develop materials and structures that are ever smaller. Some of this engineering is to simply do more with less while the science of nanomaterials allows new materials to be produced with a novel range of physical and chemical properties due to the small length scales of the microstructural features of the materials. Currently, nanoscale materials have been produced with a diverse set of useful properties and can be found in common substances like sunscreen or technologically advanced microelectronic devices. A complete understanding of materials is based on knowledge of the processing used to produce an interesting material coupled with a full characterization of the structure that results. It is this structure/property relationship that is the basis of understanding any new material developed at all length scales. The extremely short microstructural length scales involved in nanomaterials and structures requires require advanced microscopy for full characterization. Electron microscopy has become an essential tool for the characterization of nanostructures. In addition, the use of focused ion beam tools and nanomanipulators now allows one to visualize and manipulate nano-materials and structures.

Characterization of nanomaterials and structures requires a broad range of electron microscopy techniques. At Sandia National Laboratories, a full range of electron microscopy techniques are applied to the characterization of nanomaterials. The techniques include scanning electron microcopy (SEM), transmission electron microscopy (TEM) and focused ion beam (FIB) imaging and micro-machining. Each one of these techniques allows some aspect of nanostructures to be explored. For instance, a modern high

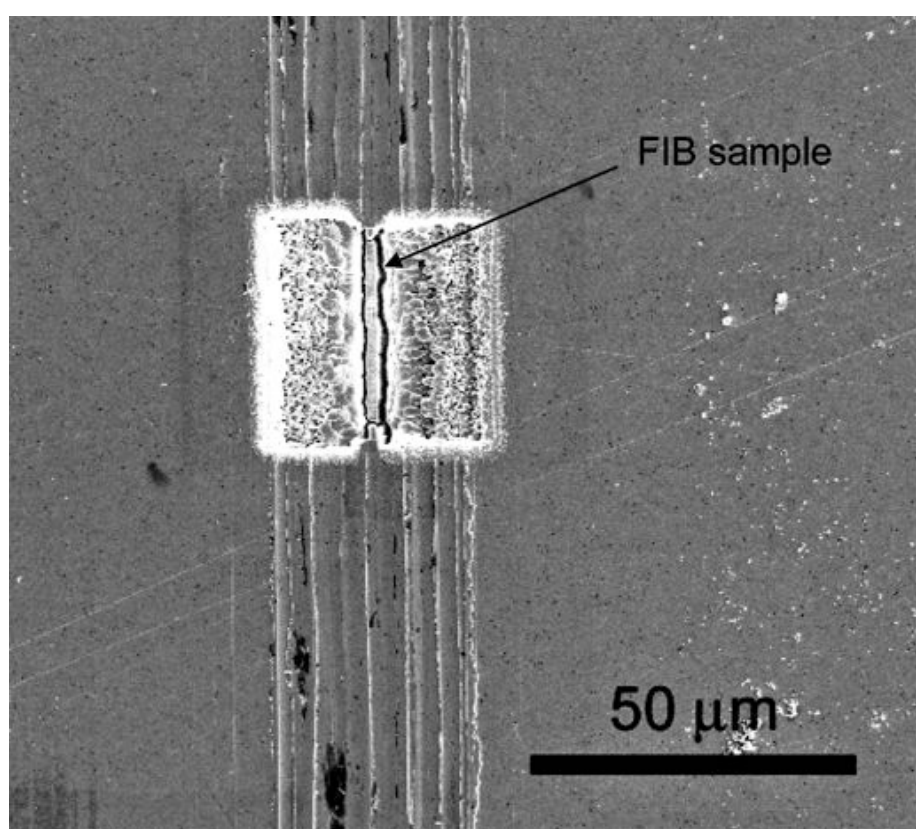

Figure 1. SEM image of a wear scar produced in electrodeposited $\mathrm{Ni}$ and the position of a FIB cross section. resolution SEM can image a surface with a resolution of about 1 $\mathrm{nm}$, obtain crystallographic information through electron backscatter diffraction (EBSD) at a $10 \mathrm{~nm}$ resolution and obtain chemical information through energy dispersive $\mathrm{x}$-ray spectroscopy (EDS) with a spatial resolution of $100-1000 \mathrm{~nm}$. TEM, and scanning transmission electron microscopy (STEM), provide high resolution imaging at a resolution as high as $0.1 \mathrm{~nm}$ and elemental information through either EDS or electron energy loss spectroscopy at resolutions of about $2-100 \mathrm{~nm}$. Focused ion beam tools are becoming essential to the study of nanomaterials due to the ability to both prepare excellent samples for TEM and SEM through micro-machining and to manipulate and actually produce nanostructures through ion beam induced deposition (IBID). It is the objective of this article to demonstrate the applications of these techniques to a range of nanomaterials and structures. An excellent detailed review of microscopy and microanalytical techniques for nanoscale materials characterization is available. ${ }^{1}$

An extremely important aspect of microscopy and microanalysis applied to nanomaterials is the synergism that can exist between the various techniques normally employed for characterization. This is obvious for the case of FIB instruments that are equipped with both SEM and FIB columns for imaging and ion beam micromachining. The ability to image with the instrument's SEM column, while micromachining with the instrument's FIB column, is invaluable for the preparation of TEM samples. When the FIB tool is also equipped with EDS and EBSD detectors, the instrument becomes a very powerful nano-characterization tool. In the same way it is extremely important in a modern characterization laboratory to have immediate access to SEM, FIB, and TEM instruments. Once all of these tools are readily available for use, it is amazing what integrated information can be obtained. Another way to look at this synergism is to consider how one might perform multiple characterization techniques on the same area of the same sample. FIB sample preparation provides a sample, that may be small, but that is amenable to study via multiple techniques like SEM, EBSD and TEM or STEM. In this way the bulk microstructure, chemistry

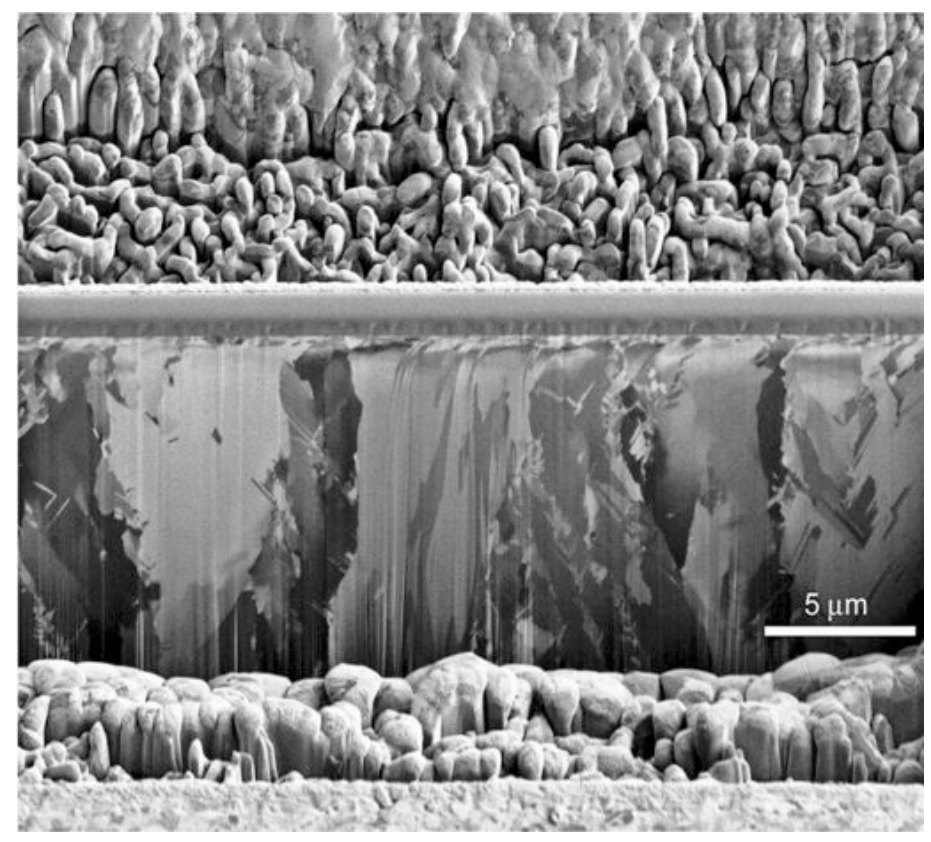

Figure 2. Ion channeling image of the deformation under a wear scar caused by a $10 \mathrm{~g}$ load slid across the area 1000 times. The sliding direction is left to right. 


\section{It's Difficult To Do A Side-By-Side Comparison When Your Coater Stands Alone.}

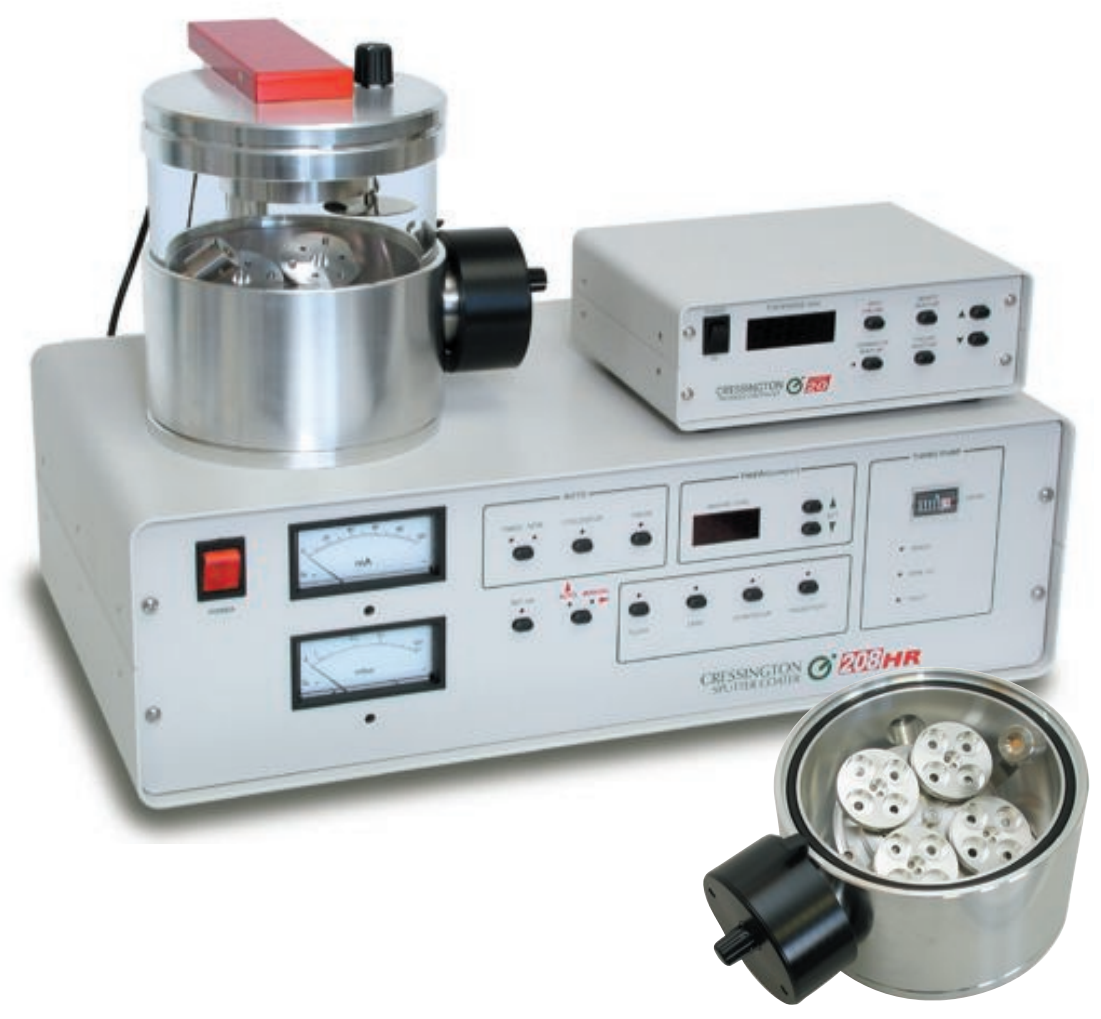

\section{High Resolution Sputter Coater 208HR for FE-SEM}

\section{Superior Features:}

- Wide Choice of Coating Materials

- Precision Thickness Control

- Multiple Sample Stage Movements

- Variable Chamber Geometry

- Wide Range of Operating Pressures

- Compact Modern Benchtop Design

\section{○ TED PELLA, INC.}

Microscopy Products for Science and Industry
4595 Mountain Lakes Blvd., Redding, CA 96003-1448

Phone: 530-243-2200 or 800-237-3526 (USA) • FAX: 530-243-3761

Email: sales@tedpella.com 
and crystallography of the specimen can be correlated with more detailed information obtained from TEM or STEM techniques. A number of interesting examples have been selected to illustrate the synergism and the use of multiple techniques to characterize nanoscale materials and structures.

\section{Understanding wear induced microstructural changes in electrodeposited metals}

Electrodeposited metals, such as $\mathrm{Ni}$, have been used to produce small parts for micro-electromechanical systems (MEMS). These parts are produced by the electrodeposition of the metal into lithographically prepared molds. In order to use these parts in critical assemblies it is important to fully understand the materials response to tribological contact particularly at the micro- and nano-length scales. Due to the small size of the electrodeposited parts and the low normal loads that are necessary for testing, a special wear tester has been developed. This system can apply very small loads from $1 \mathrm{~g}$ to $100 \mathrm{~g}$ and performs the wear test by unidirectional movement of a counterface against the electrodeposited metal part. The wear scars produced at the light loads used in this study result in narrow wear scars that may be as wide as $50 \mu \mathrm{m}$ or less. The objective of this study was to characterize the degree of subsurface deformation that accompanies wear and to assess the ability of coatings to limit the subsurface deformation and damage. ${ }^{2}$

Typical mechanical cross sectioning of the wear scars is difficult or impossible due to the small size of the wear scars and the limited depth of damage. However, FIB cross sections are a straight forward way to obtain site-specific cross sections of the wear scar and these cross sections are perfect for analysis by SEM and EBSD to assess the depth of damage and STEM to image the nanoscale grain region that forms at the surface of the sample. Figure 1 shows a typical wear scar on a $\mathrm{Ni}$ electro deposit produced using a $\mathrm{Si}_{3} \mathrm{~N}_{4}$ ball and a normal load of $10 \mathrm{~g}$. Ion channeling contrast imaging of the grains in the FIB provides a qualitative view of the deformation and is shown in figure 2 . The wear induced deformation has resulted in the shearing of the grains as they intersect the wear scar.

Electron backscattered diffraction (EBSD) in the SEM can be used to quantitatively map out grain orientations. EBSD is accomplished by moving the electron beam in a pixel-by-pixel manner over the area to be analyzed. At each pixel a diffraction pattern

a

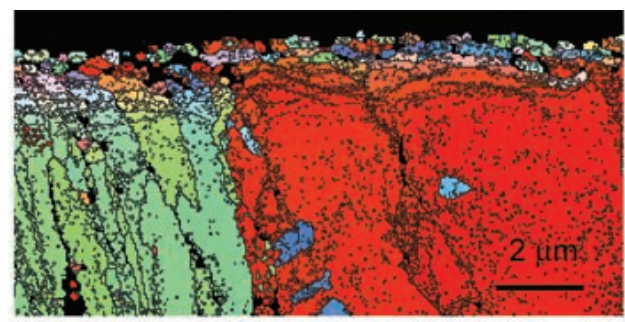

b
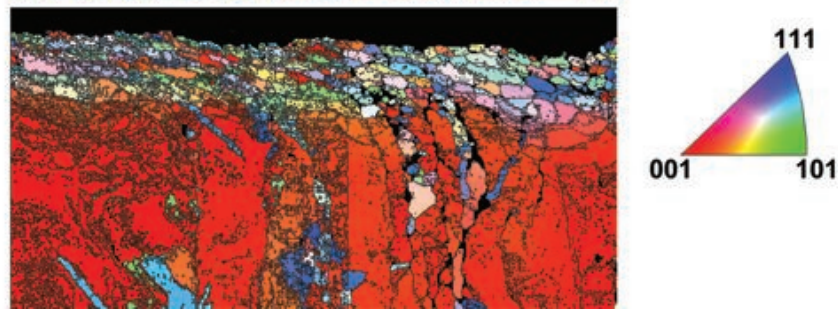

Figure 3. Orientation maps with respect to the surface normal of wear scars resulting from $10 \mathrm{~g}$ and $100 \mathrm{~g}$ loads for 1000 cycles. Note the increased depth of deformation for the $100 \mathrm{~g}$ case. a) $10 \mathrm{~g}$ for 1000 cycles, b) $100 \mathrm{~g}$ for 1000 cycles. Color key is shown at right.

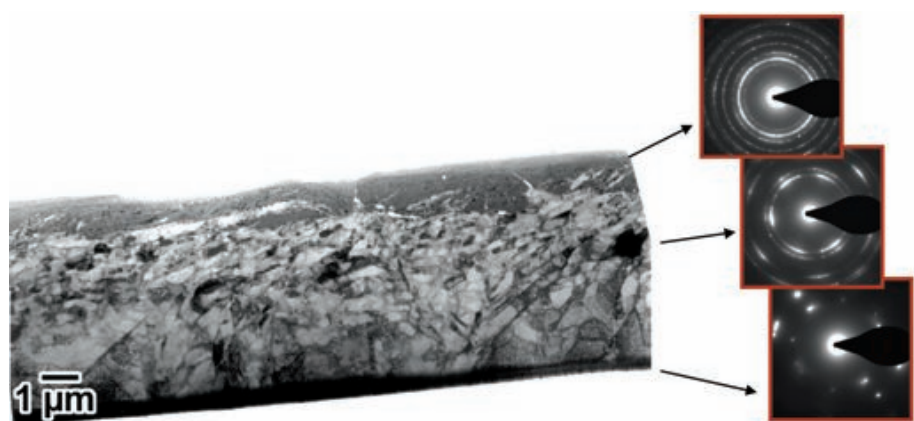

Figure 4. TEM bright field image and SAED patterns from electrodeposited Ni after wearing with a load of $100 \mathrm{~g}$ for 1000 cycles. is collected and indexed and the orientation of the pattern with respect to some relevant frame of reference (in this case, the wear direction) is determined. The orientations are then color keyed with respect to a stereographic triangle and an orientation map is produced. Current technology allows as many as 100 patterns per second to be analyzed. Figure 3 shows orientation maps obtained from electrodeposited $\mathrm{Ni}$ after wear testing with loads of $10 \mathrm{~g}$ and $100 \mathrm{~g}$ for 1000 cycles. It is readily apparent that the $100 \mathrm{~g}$ load results in much more wear induced shearing and also causes a fine-grained region at the surface to form. However, further examination of the $100 \mathrm{~g} 1000$ cycle sample in TEM shows the formation of an unsuspected nanocrystalline layer at the surface. Figure 4 shows a TEM bright field image and selected area electron diffraction patterns of the sample from which the orientation maps were obtained. The TEM images along with the diffraction patterns demonstrate that the wear has produced three distinct layers in the sample. The first layer is the nanostructured material that is located at the wear surface. SAED results in a ring pattern indicating that the layer is nanocrystalline. Below this layer is a deformed layer of larger grains followed by an undeformed region. The later two regions were evident in the EBSD orientation maps while TEM was required to prove the existence of the nanocrystalline region.

Tribological coatings are often used to reduce wear induced damage. Figure 5 shows ion channeling images and EBSD orientation maps that show the results of wear testing at a range of normal loads of diamond-like carbon thin films on electrodeposited $\mathrm{Ni}$. Figure 5a demonstrates that there is little or no wear-induced, sub-surface deformation of the diamond-like carbon coated sample at a normal load of $10 \mathrm{~g}$. At a load of $50 \mathrm{~g}$ (Figure 5b), the beginnings of sub-surface damage is evident by the slight shearing of the grains near the surface and the changes in orientation of the grains

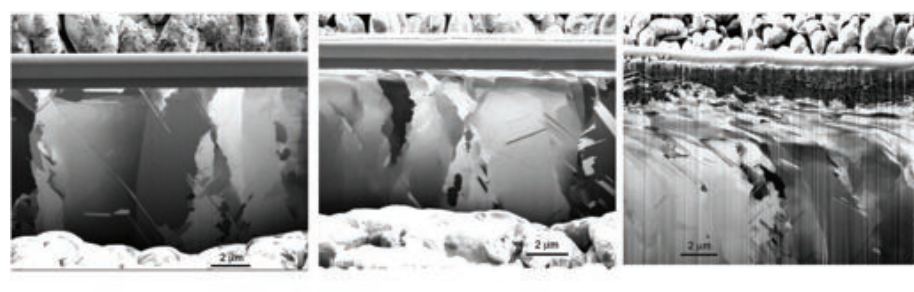

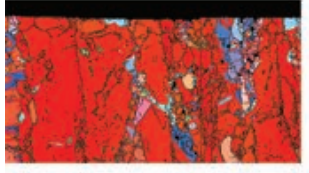

a.

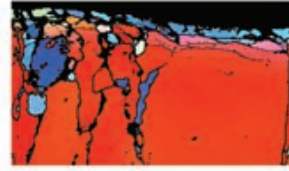

b.

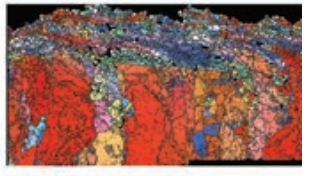

Figure 5. Channeling contrast imaging and EBSD demonstrates ability of diamond like carbon to prevent wear induced sub-surface damage. a) $10 \mathrm{~g} 1000$ cycles, b) $50 \mathrm{~g} 1000$ cycles and c) $100 \mathrm{~g} 1000$ cycles. 


\section{Value and Excellence in SEMs}

\section{VEGA Scanning Electron Microscopes}

Fully automated PC-controlled SEMs designed for a multi-user environment.

High performance 4-lens optics provides high resolution, high depth of focus, extremely large field of view as well as optimum working conditions for any application.

High working vacuum and variable pressure operation up to $2000 \mathrm{~Pa}$.

Original patented Low Vacuum Secondary Detector for true secondary electron imaging under low vacuum.

Full range of precision-engineered specimen chambers and stages.

Sophisticated yet easy-to-use software for microscope control and image processing \&t evaluation.

Network operations with built-in remote control and diagnostics.

3D surface reconstruction using $3 \mathrm{D}$ beam technology.
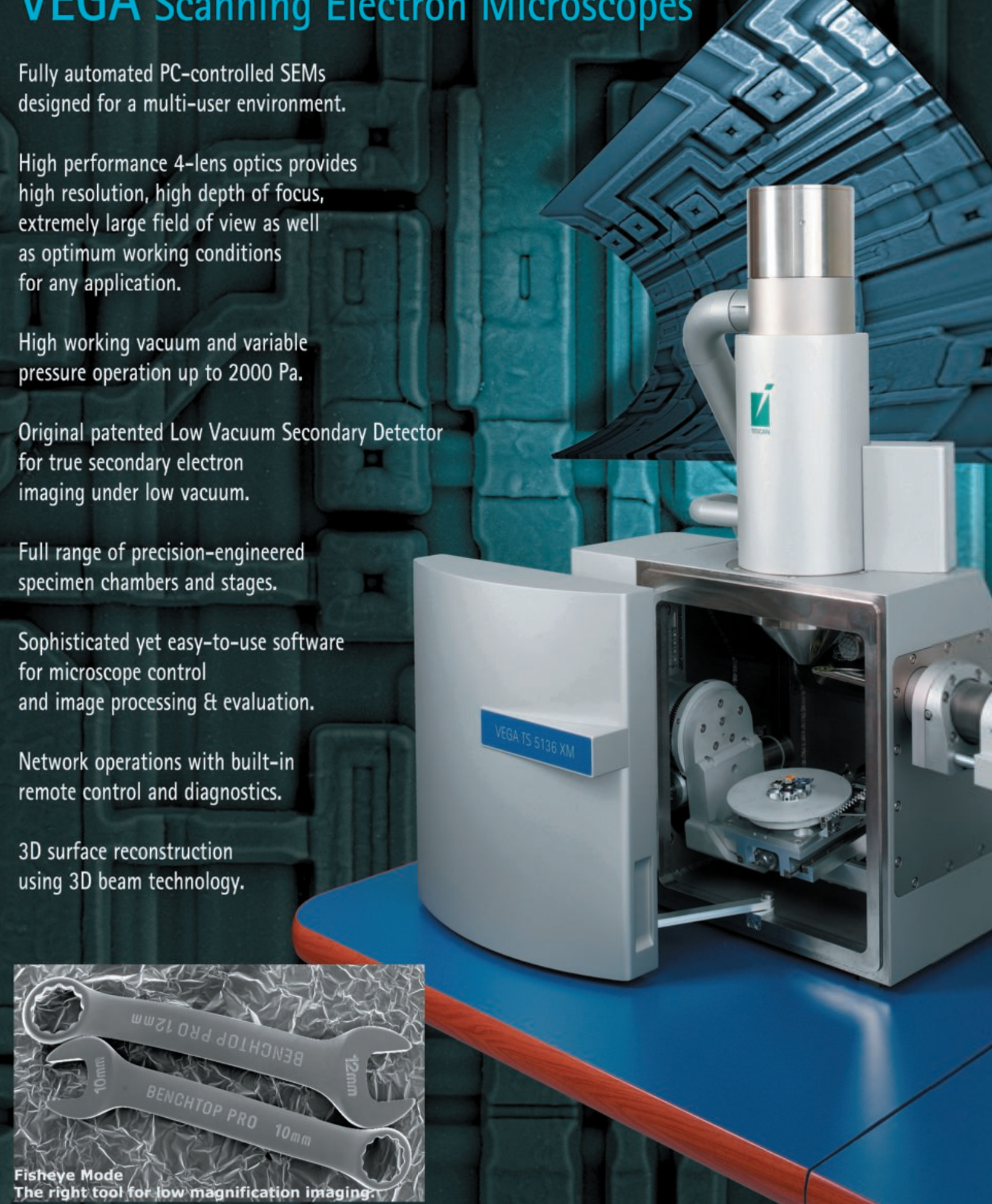

西


in the EBSD map. Wear testing at a normal load of $100 \mathrm{~g}$ (Figure 5c) has resulted in an extreme amount of sub-surface damage and the failure of the diamond-like carbon layer. This is most likely a result of delamination from the substrate and subsequent fracture of the diamond-like carbon layer, which then acts to cause more extensive damage to the substrate.

At this time it is difficult to predict friction coefficients and subsurface damage due to sliding contact. Complete characterization of wear induced sub-surface damage will improve our understanding of the wear phenomenon. Combined use of characterization techniques, including SEM, FIB, EBSD and TEM, are required to obtain necessary information and can provide new insights into wear and friction and the effect of tribological coatings as shown here.

\section{Understanding nanoscale void nucleation and growth dur - ing pitting corrosion of aluminum}

Corrosion is an important degradation phenomenon in many materials systems and applications. Many metals rely on a thin, adherent metal oxide coating to passivate surfaces and improve the corrosion resistance of the metal. Generally, localized corrosion of passivated metals starts at structural or compositional flaws in the passive oxide, which then progresses to rapidly propagating structures like pits. One process under current study that might prove to be responsible for pit initiation is the nucleation and growth of nanoscale voids. ${ }^{3}$ Nanoscale processes in the thin passive oxide layers are normally invoked to describe the nucleation and growth of voids. Electron microscopy has proven useful in the understanding of the initial stages of pit nucleation and growth at the nanoscale of $1-10 \mathrm{~nm}$. An ability to detect and study how nanoscale voids nucleate and grow under electrochemical conditions is a valuable tool to determine the role of voids in the nucleation of localized corrosion.

Figure 6 shows high resolution SEM images of the surfaces of $\mathrm{Al}$ test coupons that were anodically polarized at $5 \mathrm{nA} / \mathrm{cm}^{2}$ for progressively longer times. Note that the number of voids or pores increases $(1000 \mathrm{sec})$ and then the pores or voids begin to grow into each other at longer times. Although high resolution SEM imaging can show that there are either voids or pores present, it cannot distinguish between the two. Cross sections through polarized surfaces for TEM and STEM analysis were prepared using FIB. In this case, the use of a two column FIB (electron and ion) allowed
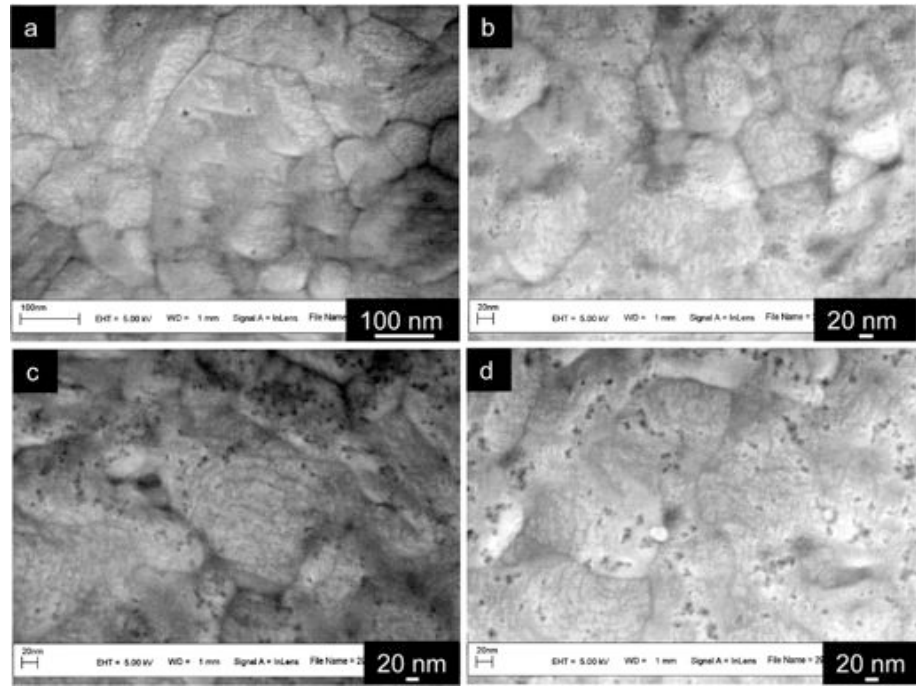

Figure 6. SEM images of Al after polarization showing the progression of void nucleation and growth a) $0 \mathrm{sec}$., b) $1000 \mathrm{sec}$., c) $2000 \mathrm{sec}$., and d) $4000 \mathrm{sec}$.
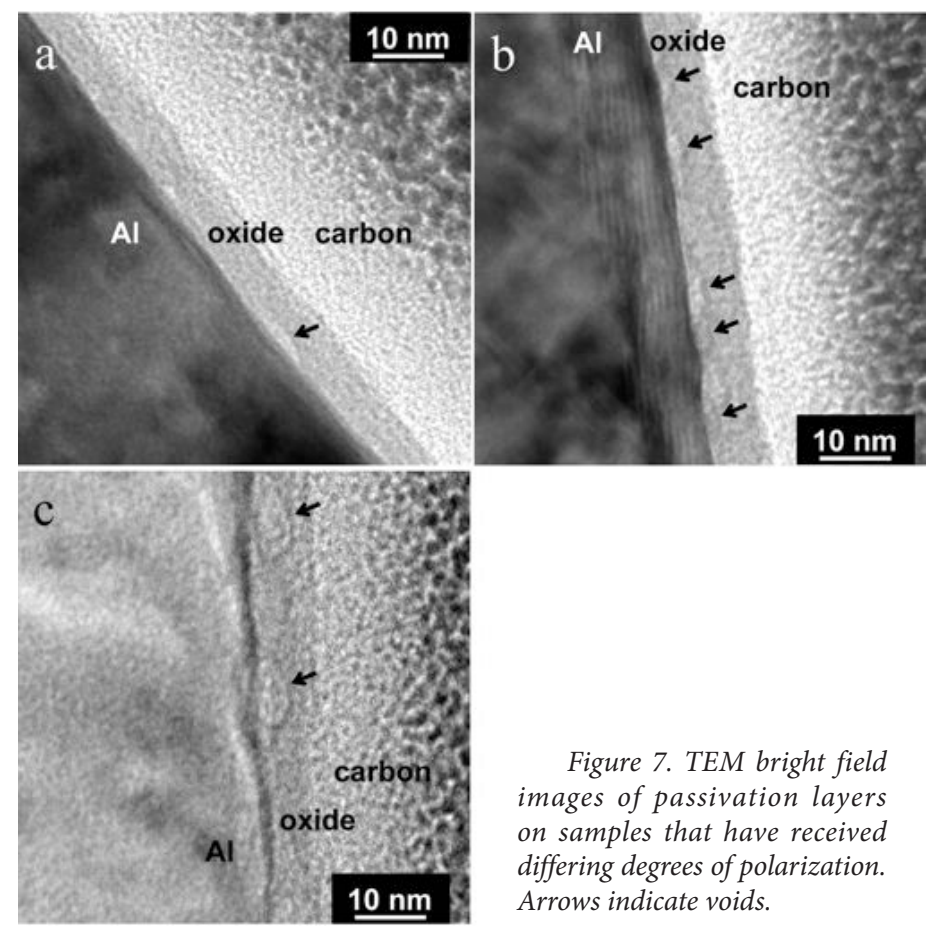

Figure 7. TEM bright field images of passivation layers on samples that have received differing degrees of polarization. Arrows indicate voids.
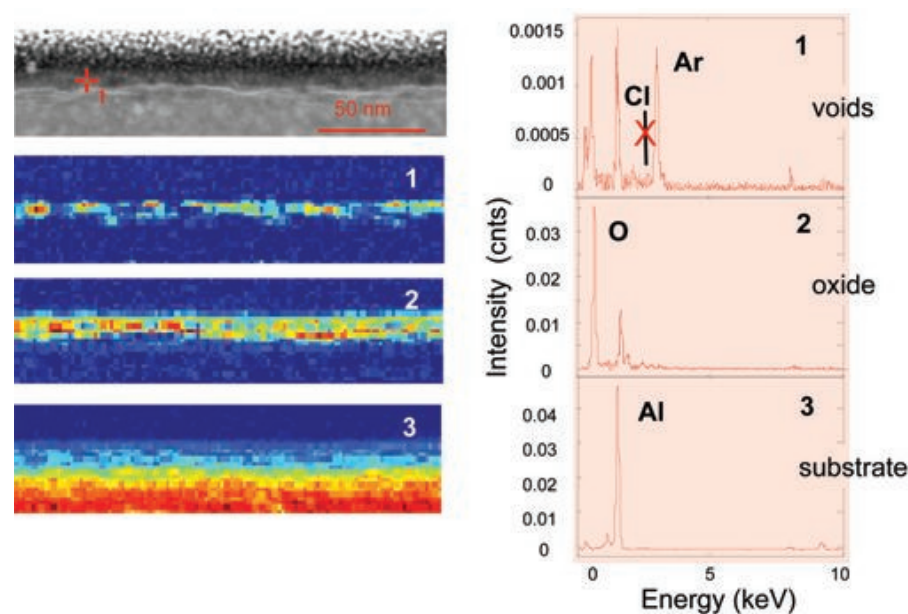

Figure 8. STEM x-ray microanalysis of voids in oxide films on Al. There is no $\mathrm{Cl}$ associated with the voids in the oxide layer.

the cross section to be precisely placed with respect to the surface features. Bright field TEM images of the passivation layers are shown in Figure 7. The samples were prepared by first evaporating a heavy carbon layer on to the samples to protect the passivating oxide layers. This was followed by standard FIB-TEM preparation techniques. Figure 8 shows the results of $x$-ray spectral imaging of the oxide layer. The STEM annular dark field image shows the voids as lighter regions. The $\mathrm{x}$-ray result shows that the voids con-

a
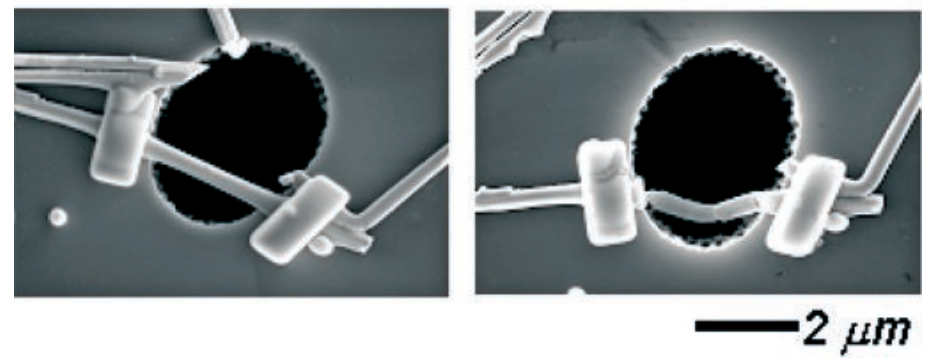

Figure 9. Micrograph images of Au nanowire (a) before and (b) after being subject to indentation. 
tain Ar remaining from cleaning of the films, but no $\mathrm{Cl}$ signal. The lack of $\mathrm{Cl}$ in the voids shows that the voids are not precursors for microcorrosion cells, as postulated by one model. The techniques of SEM, TEM and STEM with x-ray microanalysis have provided new insights into localized corrosion of $\mathrm{Al}$. This knowledge may allow the initiation of localized corrosion to be understood and subsequently controlled. 3

\section{Manipulation of Nano-wires for micromechanical testing}

The measurement of the mechanical properties of nanowires is of current interest. ${ }^{4}$ However, the small size of nanowires makes the placing and holding of the nanowires very difficult. The goal of our work here was to place nanowires composed of either Au or $\mathrm{ZnO}$, over appropriate openings or on microtesting devices, fix the wires in place, and perform a mechanical characterization. A 3-axis computer controlled micromanipulator installed in the FIB sample chamber was used to move the nanowires into position, followed by $\mathrm{Pt}$ deposition to hold the wires in place. Mechanical deformation of Au wires, including dislocation mediated plasticity, may be examined when wires are first fixed in place over a through-hole via and then deflected using a nanoindenter machine. Figure 9 shows a gold nanowire fixed in place over a hole (a) before and (b) after it has been indented using a spherical indenter tip. The quantitative results of the nanowire bending test are shown in Figure 10. Based on the cross-sectional geometry shown in the inset of Figure 10, the stress/strain plot is determined from the load/displacement data measured using the nanoindenter machine. Key results include the material modulus values 54-62 GPa (obtained from the slope of the unloading curves), the yield stress $\sim 120 \mathrm{MPa}$, and the maximum

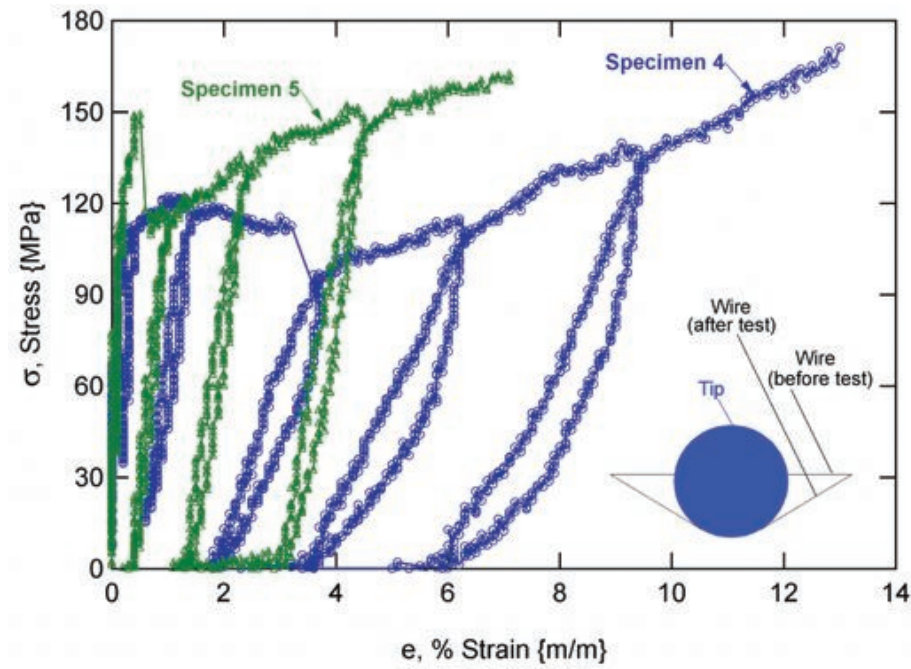

Figure 10. Stress/strain plot for Au nanowire specimens. The inset identifies the assumed cross-sectional geometry used in the analysis.

strain at failure $\sim 13 \%$. Also noteworthy is that the wire itself must be scanned using the indenter tip to accurately obtain the location of the specimen prior to indentation.

More elaborate techniques have been used to pick and place individual wires into a MEMS enabled tensile testing machine. Recall, the FIB machine (a) is equipped with a 3 -axis controlled probe tip, (b) is capable of user-defined Pt deposition, (c) is capable of user-defined cutting operations, and (d) is equipped with a 5-axis sample stage. As shown in Figure 11, these capabilities may be used to locate, collect, orient and then attach a wire into a designated location on the MEMS enabled tensile testing device.
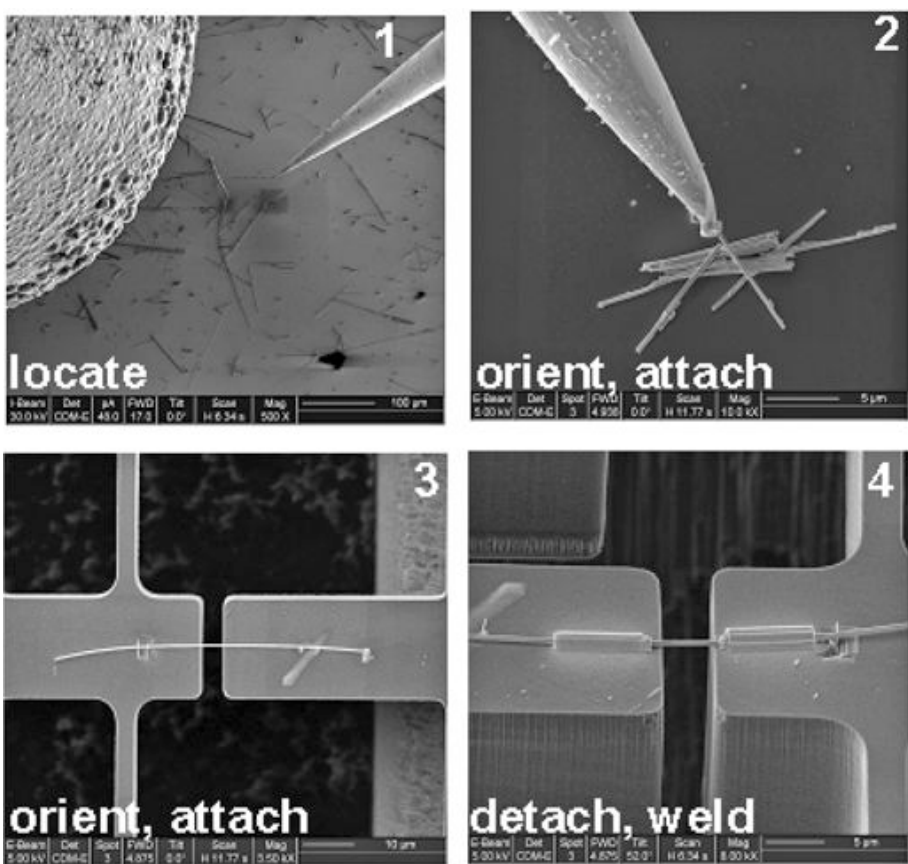

Figure 11. Pick and place attachment sequence for nanowire specimens. The sequence of operations is used to select and locate specimens into the designated location in a MEMS enabled tensile test machine.

Successful placement of both $\mathrm{Au}$ and $\mathrm{ZnO}$ nanowires is demonstrated in Figure 11 that shows nanowires fixed into the designated

a
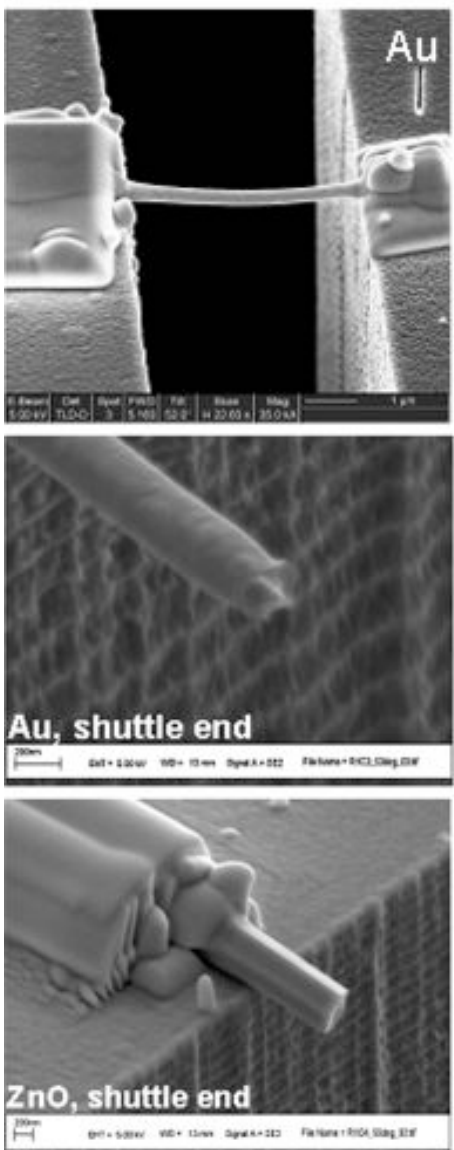

Figure 12. Successful placement of (a) Au and (b) $\mathrm{ZnO}$ nanowires into designated location on MEMS tensile test machine.

Figure 13. Fractography images for both ends of Au nanowire.

Figure 14. Fractography images for both ends of $\mathrm{ZnO}$ nanowire. 


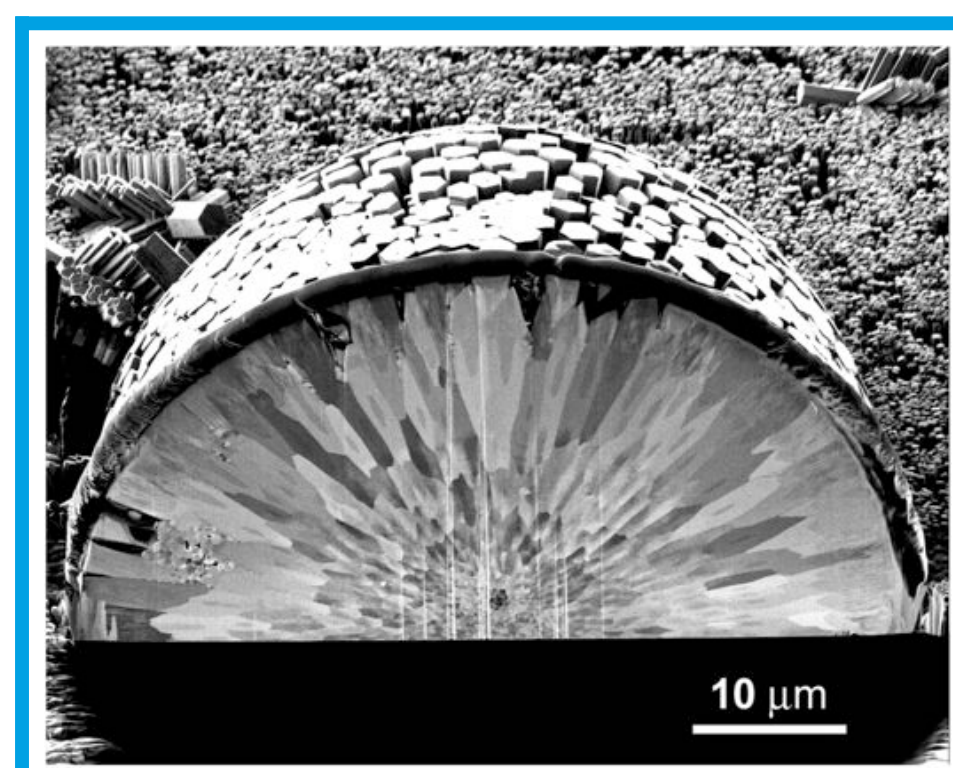

Figure 15. FIB prepared cross section of $\mathrm{ZnO}$ dome structure.

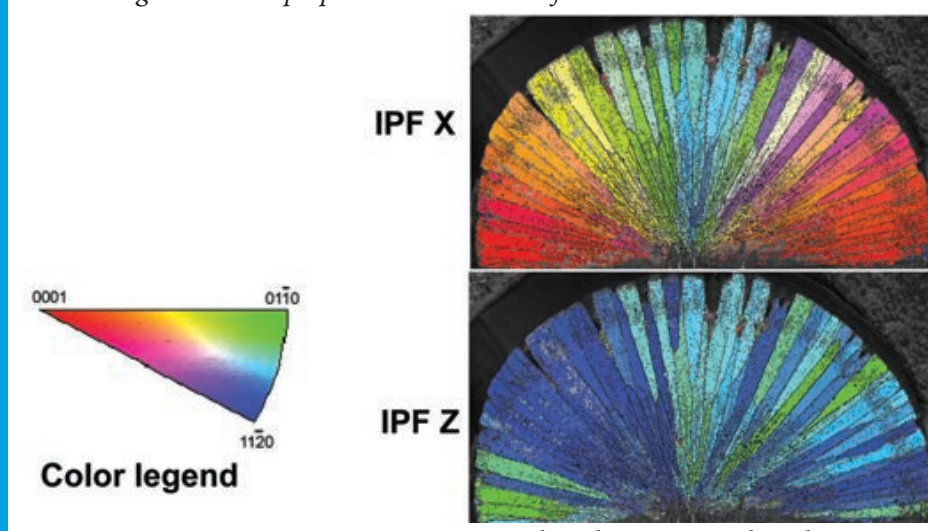

Figure 16. EBSD orientation maps that demonstrate that the $\mathrm{ZnO}$

domes are formed from individual columnar grains that have a basal plane growth direction.

location in the MEMS tensile test machine. Once in place, the wires may be tensioned using an actuator integrated into the test machine. The resulting fracture surfaces are shown in Figure 12 and Figure 13, for $\mathrm{Au}$ and $\mathrm{ZnO}$ nanowires respectively. The Au specimen, Figure 12, demonstrates a cone/cone morphology, appearing similar to the shear glide mechanism, typical of macroscopic single crystals. The $\mathrm{ZnO}$ specimen, in Figure 13, demonstrates a brittle cleavage failure occurring across the c-plane. Current research efforts focus on the accurate determination of stress and strain for nanowire specimens up to failure.

\section{Understanding Growth of ZnO Hemispherical Particles}

$\mathrm{ZnO}$ is an excellent oxide material for determining how small crystals with specific morphologies and orientations may be produced. One set of conditions produced hemispherical assemblages of $\mathrm{ZnO}$ crystals. To understand the development of these features, the FIB was used to produce a cross section through one of the dome-like structures. Figure 15 is an ion channeling image taken with a $30 \mathrm{kV} \mathrm{Ga}^{+}$ion beam that shows the columnar grain structure present in the dome-like $\mathrm{ZnO}$ structure. Following this, a TEM section was prepared by FIB milling away the back side of the sample. The thin sample was then lifted-out ex-situ and mounted on a carbon coated TEM grid. The TEM grid with the FIB prepared sample was mounted in the SEM at the appropriate tilt for EBSD orientation mapping. The EBSD maps are shown in Figure 16 along with the stereographic triangle color key. Interpretation of EBSD maps from structures like the $\mathrm{ZnO}$ domes are a bit more complicated than for other more conventional materials. Here, the direction within the dome must be considered when viewing the maps in Figure 16. However, if one simply considers the map with respect to the $\mathrm{X}$-direction, then we see that any grain closely aligned with the X-direction is colored red. This indicates that grains with a basal plane alignment of the hexagonal crystal structure. Of additional interest is that there are only two rotational alignments of the normal to the growth direction as shown by the map with respect to the Z-direction as we see only two colors in this map. Further investigation of this sample with TEM indicated why the dome structures formed. At the apex of the dome, contaminate particles were found that nucleated the domes and caused the grains

A
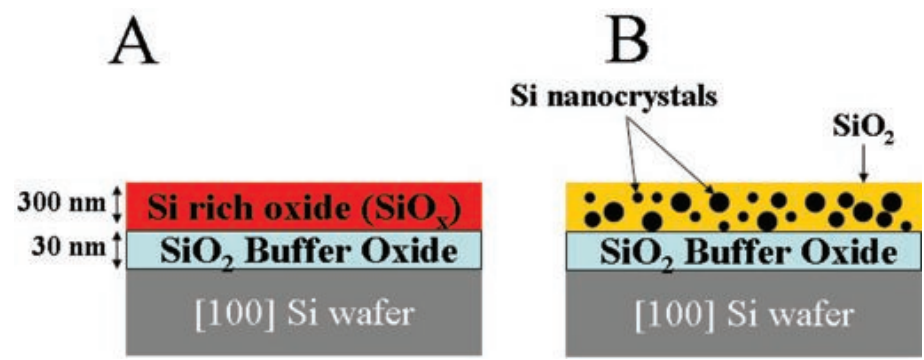

Figure 17. Schematic of Silicon nanocrystal growth from annealing of High Density-CVD SiOx films.

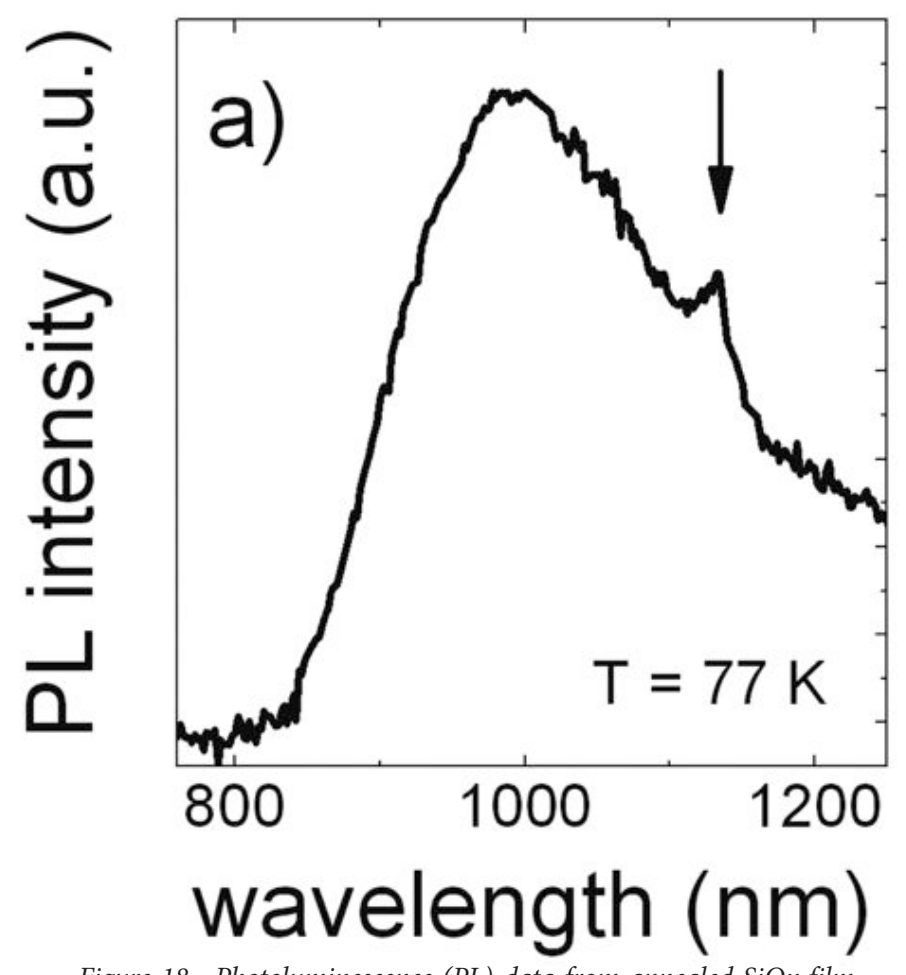

Figure 18. Photoluminescence (PL) data from annealed SiOx film. The arrow denotes the PL peak for bulk silicon.

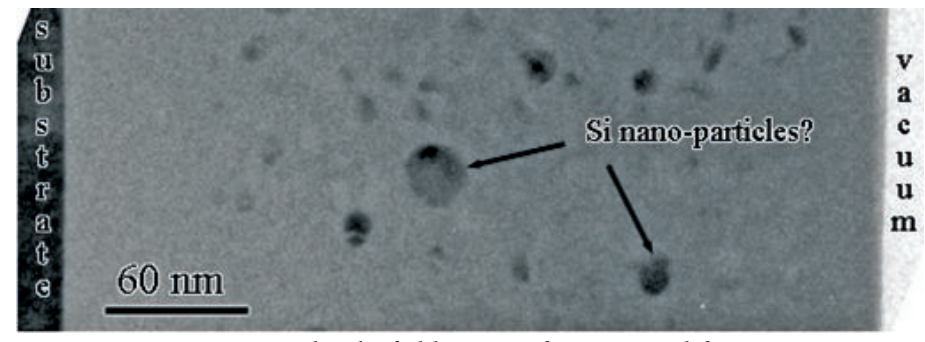

Figure 19. TEM bright field image of nanocrystal formation in an annealed film. 


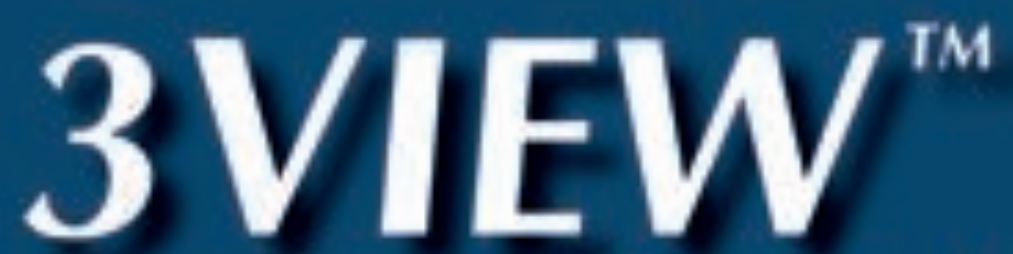

\section{Perfectly aligned image stacks}
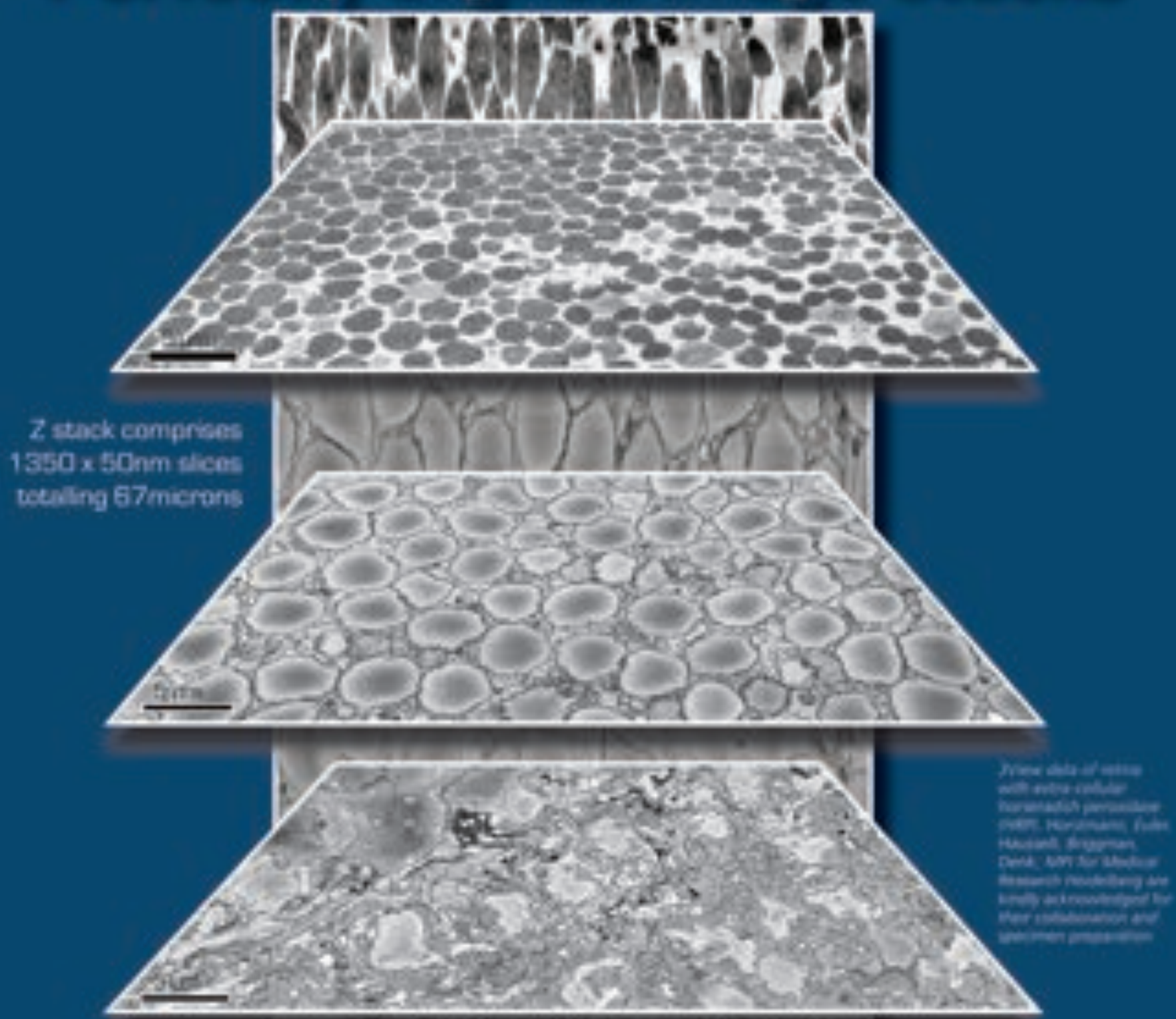

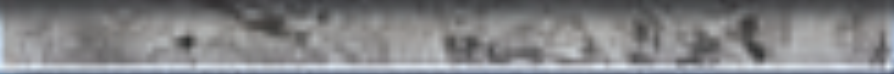

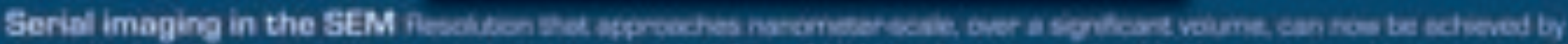

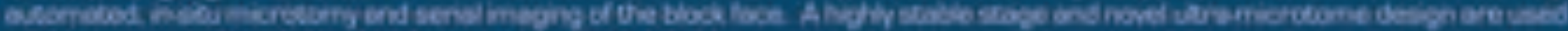

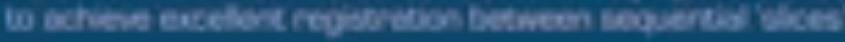

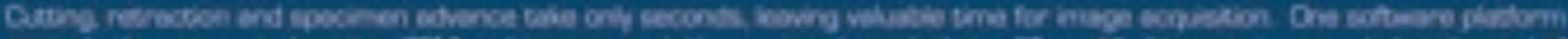

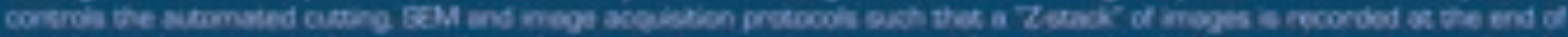

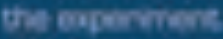

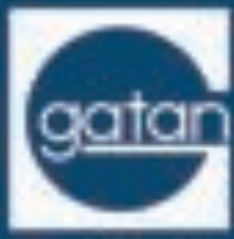

Ploose wet us at Numscience 2006 in Baceh 914

\section{A Revolution in 3D Microscopy}




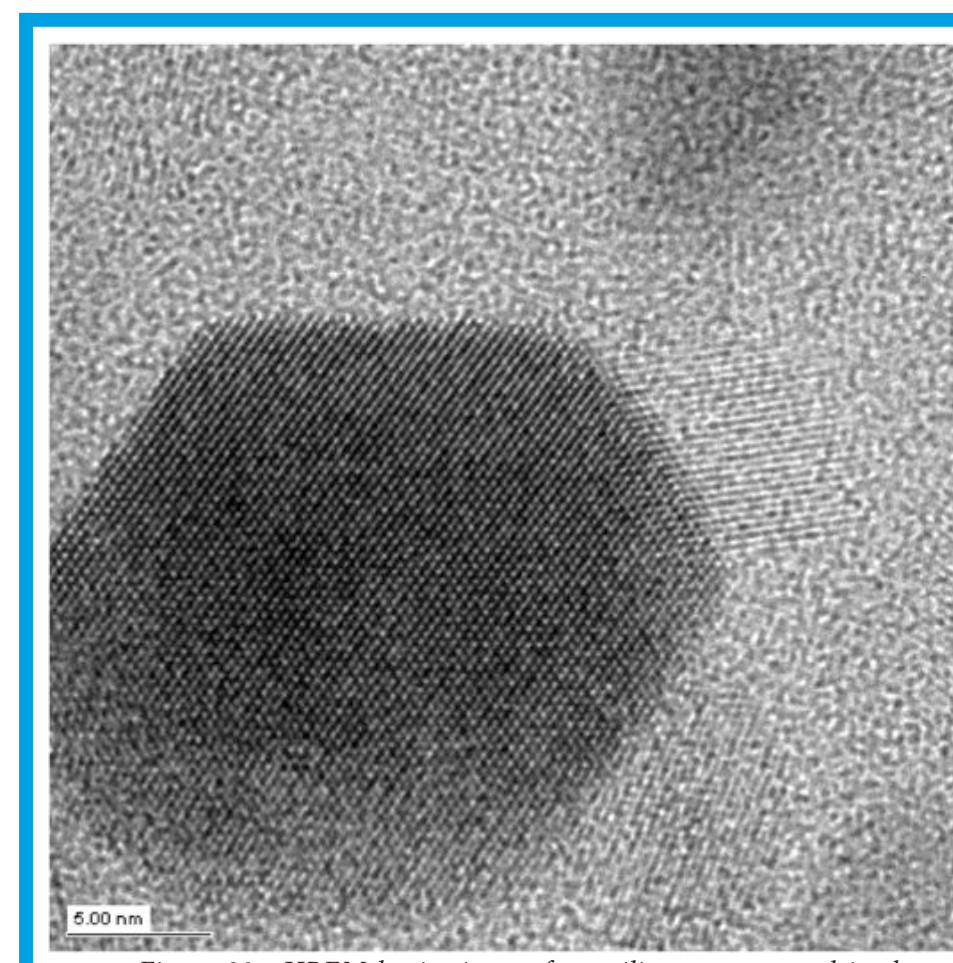

Figure 20. HREM lattice image from silicon nanocrystal in the annealed film. The lattice fringe spacing is $3.1 \AA$, the spacing for the $\{111\}$ planes in silicon.

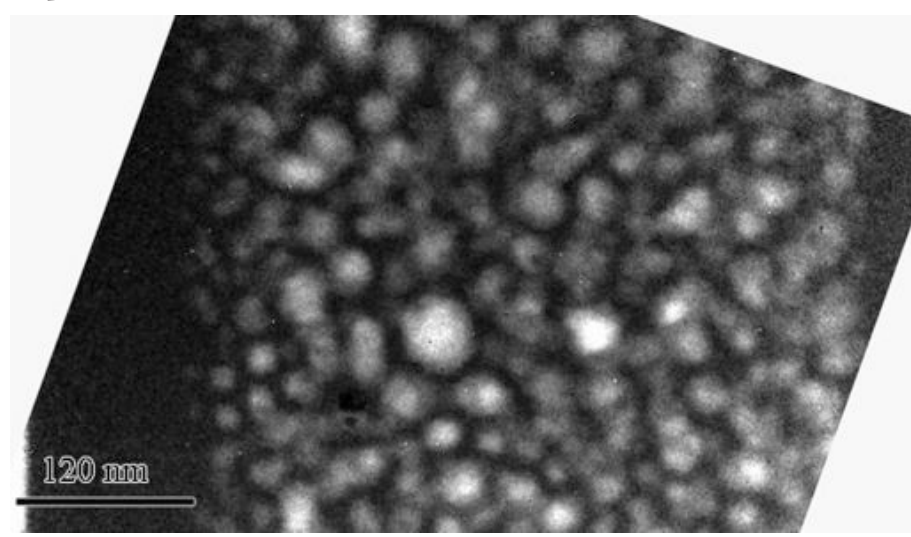

Figure 21. Si jump ratio image from the annealed film. This image shows the density and size distribution of silicon particles. Note the denuded region on both sides of the film.

to radiate out from the origin particle. This knowledge allowed better control of the growth of the $\mathrm{ZnO}$ domes.

\section{Si nanoparticles in $\mathrm{SiO}_{\mathbf{x}}$ produce unique Photolumines- cence}

A key area of technical interest for nanoscale materials is quantum confinement. The electroptical properties of materials at the nanoscale are measurably different from those in the bulk. While the ability to tune these properties at the nanoscale is attractive, the necessary process control to manipulate materials at this length scale remains elusive. The application of precipitation kinetics and thermodynamics has been used to investigate quantum confinement effects in silicon $(\mathrm{Si})$ nanocrystals in a silica $\left(\mathrm{SiO}_{\mathrm{x}}\right)$ matrix, shown schematically in Figure 17..$^{\text {This }}$ process consists generally of depositing a Si-rich, SiOx film onto a Si substrate and then annealing this film at elevated temperatures to precipitate $\mathrm{Si}$ nanocrystals. Films treated in this manner have been shown to exhibit optical properties that are different from the bulk. Figure 18 shows photoluminescence (PL) data from an annealed film that produces a broad, blue-shifted peak at $\sim 1000 \mathrm{~nm}$. Typical bulk silicon shows a characteristic peak at $\sim 1130 \mathrm{~nm}$ at $77 \mathrm{~K}$.

In order to connect these optical measurements with the predicted nanostructures, TEM was performed on conventionallyprepared, cross-sectional samples of the annealed films. The bright field image in Figure 19 shows the overall structure of the film. A region in the center of the film shows some sort of particles with portions of the film on either side without any apparent particles. However, the image in Figure 19 does not help in determining the exact characteristics of these nanoparticles.

High resolution electron microscopy and energy filtered imaging were used to fully characterize the Si nanoparticles. High resolution electron microscopy (HREM) was used to identify the structure of the particles. Lattice images from several particles had measurable lattice fringe spacings which corresponded closely to the $3.1 \AA\{111\}$ spacing of silicon. In addition, the particle in Figure 20 shows clear facets. The jump ratio image in Figure 21 was formed using pre- and post-edge images from the Si L-edge at $99 \mathrm{eV}$ energy loss. The energy filtered image shows a much larger number of $\mathrm{Si}$ nanoparticles than the bright field image alone of Figure 19 would suggest. This image also clearly shows the denuded regions at both the substrate and the film surface. By combining these imaging and analytical TEM techniques, we are able to verify the Si nanoparticle structure, number density and composition in this quantum confinement experiment.

\section{Conclusions}

The examples shown here were selected to highlight the breadth of nanomaterials of interest and to highlight the absolute necessity of access to and application of complimentary microscopy and microanalytical techniques. Nanoscale materials and processes are a challenge to completely characterize even with all of the techniques discussed in this article.

\section{Acknowledgements:}

The authors would like to acknowledge Bonnie McKenzie, Michael Rye and Garry Bryant for their help with instrumentation and sample preparation. The authors would also like to thank M. S. Carroll and W. Pan for the Si nanocrystalline films and the photoluminescence data. Sandia is a multi-program laboratory operated by the Sandia Corporation, a Lockheed Martin Company, for the United States Department of Energy, under contract \#DEAC04-94AL8500.

\section{References}

1. Handbook of Microscopy for Nanotechnology, eds. N. Yao and Z. L. Wang, Kluwer Academic Publishers, New York, 2005.

2.S. V. Prasad and J. R. Michael, "EBSD studies of wear-induced subsurface regions in LIGA nickel”, Scripta Mat.,2003, vol. 48, 255-260.

3. K. R. Zavadil, J. A. Ohlhausen and P. G. Kotula, "Nanoscale void nucleation and growth in the passive oxide on aluminum as a prepitting process", Journal of the Electrochemical Society, 2006, vol. 153, B296-B303.

4. Y. Zhu, N. Moldovan and H. D. Espinosa, "A micromechanical load sensor for in-situ electron and $\mathrm{x}$-ray microscopy tensile testing of nanostructures”, Applied Physics Letters, 2005, vol. 86, 013506-1-013506-3.

5. Wei Pan, R.G. Dunn, M.S. Carroll et al., "Photoluminescence in silicon rich oxide thin films under different thermal treatments", submitted to Applied Physics Letters, 2006 


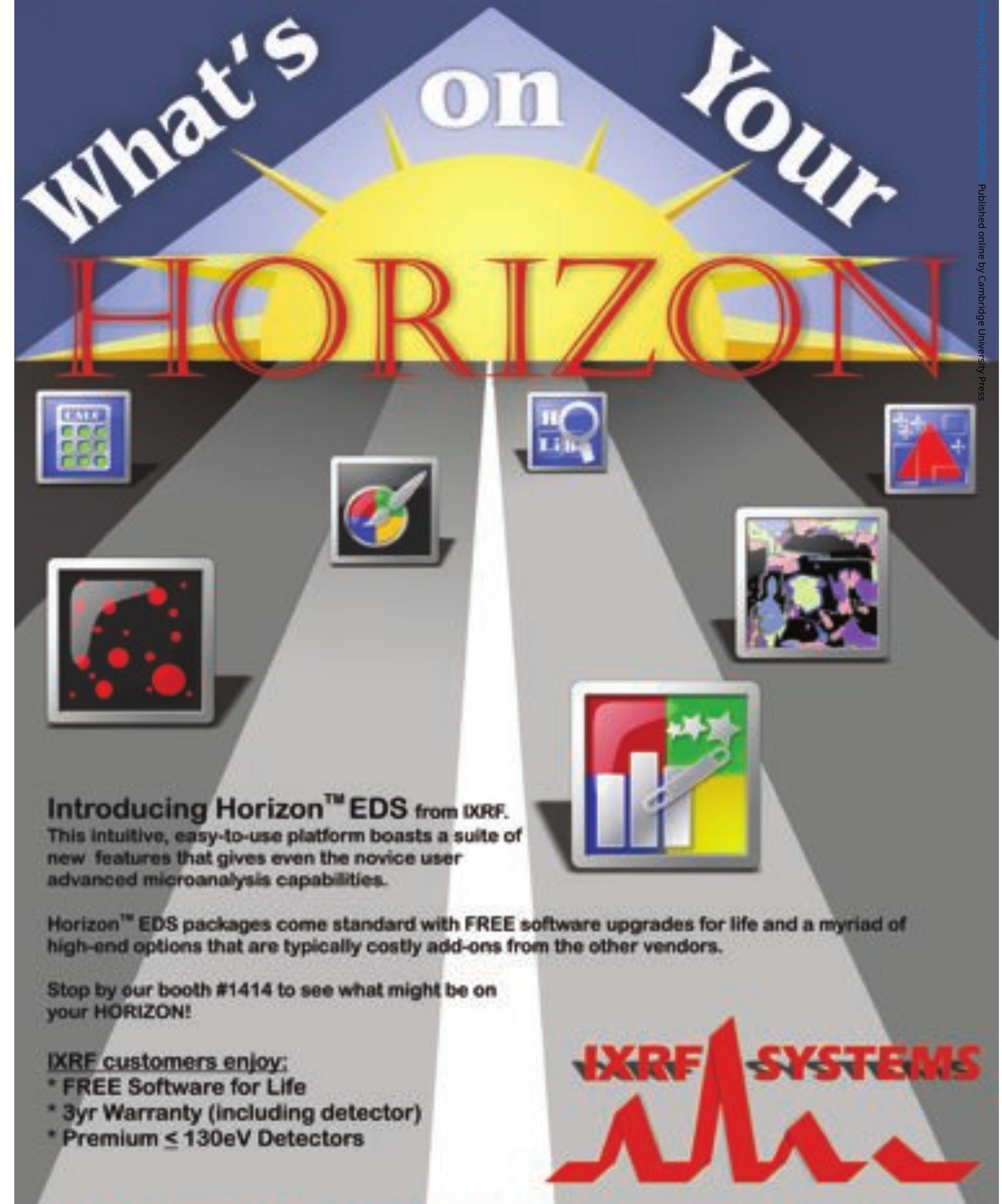

IXRF SYSTEMS, INC Houston, TX 281.286.6485 www.ixrfsystems.com 